\title{
Finite-Size Scaling of a First-Order Dynamical Phase Transition: Adaptive Population Dynamics and an Effective Model
}

\author{
Takahiro Nemoto, ${ }^{1,2}$ Robert L. Jack, ${ }^{3}$ and Vivien Lecomte ${ }^{1,4}$ \\ ${ }^{1}$ Laboratoire de Probabilités et Modèles Aléatoires, Sorbonne Paris Cité, UMR 7599 CNRS, Université Paris Diderot, \\ 75013 Paris, France \\ ${ }^{2}$ Philippe Meyer Institute for Theoretical Physics, Physics Department, École Normale Supérieure \& PSL Research University, \\ 24 rue Lhomond, 75231 Paris Cedex 05, France \\ ${ }^{3}$ Department of Physics, University of Bath, Bath BA2 7AY, United Kingdom \\ ${ }^{4}$ LIPhy, Université Grenoble Alpes and CNRS, F-38042 Grenoble, France
}

(Received 24 November 2016; published 14 March 2017)

\begin{abstract}
We analyze large deviations of the time-averaged activity in the one-dimensional Fredrickson-Andersen model, both numerically and analytically. The model exhibits a dynamical phase transition, which appears as a singularity in the large deviation function. We analyze the finite-size scaling of this phase transition numerically, by generalizing an existing cloning algorithm to include a multicanonical feedback control: this significantly improves the computational efficiency. Motivated by these numerical results, we formulate an effective theory for the model in the vicinity of the phase transition, which accounts quantitatively for the observed behavior. We discuss potential applications of the numerical method and the effective theory in a range of more general contexts.
\end{abstract}

DOI: 10.1103/PhysRevLett.118.115702

Introduction.-Systems far from equilibrium display a wide spectrum of complex behavior [1,2]. For example, thermodynamic phase transitions are usually forbidden in one-dimensional systems, but a variety of dynamical phase transitions are still observed [3-7]. Such transitions can appear in far-from-equilibrium states that are defined by restricting (or conditioning) trajectories so that timeaveraged observables take nontypical values [8]. They can be related to physical properties of systems where metastability is important, especially glassy systems [7,9-16].

In some cases these transitions can be studied analytically $[5-7,17,18]$, but in practical applications one must often resort to numerical methods: these access the relevant far-from-equilibrium states by rare-event sampling algorithms [13,19-22], employing, for instance, population dynamics or path sampling. Such methods tend to perform poorly in the vicinity of dynamical phase transitions, just as conventional sampling methods tend to fail close to equilibrium phase transitions. For the equilibrium case, advanced methods exist that solve this problem, including finite-size scaling analysis $[23,24]$ and multicanonical sampling [25-27]. For dynamical phase transitions, some progress has been made in this direction $[16,21,28]$ but accurate calculations are numerically expensive and suffer from significant finite-size effects.

Here, we analyze a dynamical phase transition [7] in the Fredrickson-Andersen (FA) model [29]. We combine a state-of-the-art numerical approach [30] with a theoretical analysis. We show that numerical results and theoretical predictions for finite-size scaling near the phase transition agree quantitatively. By combining these ingredients we obtain a full description of the transition, at a modest computational cost. The phase transition is a prototype for transitions in a range of systems $[7,13,16,31]$, so we argue that these new methods and insights have broad potential application in this field.

Model.-The 1D FA model [29] is a kinetically constrained model that consists of $L$ spins on a periodic lattice. The $i$ th spin takes values $n_{i}=0$ (down) or $n_{i}=1$ (up) and the configuration is $\mathcal{C}=\left(n_{i}\right)_{i=1}^{L}$. We define an operator $F_{i}$ that flips the state of spin $i$, so that $\mathcal{F}_{i}[\mathcal{C}]=\left(n_{1}, n_{2}, \ldots, 1-n_{i}, \ldots, n_{L}\right)$. The kinetic constraint of the model is that spin $i$ can flip only if at least one of its neighbors is up. The transition rates between configurations reflect this constraint; they are

$$
w\left(\mathcal{C} \rightarrow \mathcal{F}_{i}[\mathcal{C}]\right)=\left[c\left(1-n_{i}\right)+(1-c) n_{i}\right] f_{i}(\mathcal{C}),
$$

where $f_{i}=n_{i-1}+n_{i+1}$ enforces the kinetic constraint and $c$ is a parameter that depends on the temperature in the model [29]. The rates obey detailed balance and the model's equilibrium distribution follows a Bernoulli law $p_{\text {eq }}(\mathcal{C}) \propto c^{\sum_{i} n_{i}}(1-c)^{L-\sum_{i} n_{i}}$. Despite this trivial distribution, the kinetic constraint in the model leads to rich behavior, related to dynamical heterogeneity in glassy systems $[13,14,32]$.

Dynamical phase transitions.-We define the dynamical activity $K(\tau)=N^{K}(\tau) / \tau$, where $N^{K}(\tau)$ is the total number of spin flips during the time interval $[0, \tau]$. The phase transitions that we consider take place in ensembles of trajectories that are restricted to a given value of the activity. In the limit $\tau \rightarrow \infty$, the activity converges to its equilibrium 
value $K_{\mathrm{eq}}$ : to estimate the probability of rare trajectories with $K(\tau) \neq K_{\text {eq }}$, we consider the cumulant generating function

$$
G(s)=\lim _{\tau \rightarrow \infty} \frac{1}{\tau} \log \left\langle e^{-s \tau K(\tau)}\right\rangle,
$$

where $\langle\cdot\rangle$ denotes an ensemble average. Dynamical phase transitions are associated with singularities in $G(s)$ : they are analogous to thermodynamic phase transitions, with $G$ corresponding to the thermodynamic free energy [11] and $s$ corresponding to an intensive thermodynamic field that is used to drive the system through its phase transition. We also define

$$
\langle K\rangle_{s} \equiv-\frac{d G(s)}{d s}=\lim _{\tau \rightarrow \infty} \frac{\left\langle K(\tau) e^{-s \tau K(\tau)}\right\rangle}{\left\langle e^{-s \tau K(\tau)}\right\rangle},
$$

which specifies the dependence of the mean activity on the field $s$, analogous to the dependence of the order parameter on its conjugate field in thermodynamics.

The dynamical phase transition that occurs in the FA model separates a high-activity state [with $\langle K\rangle_{s}=O(L)$ ] from an inactive (glass) state [with $\left.\langle K\rangle_{s}=o(L)\right]$. It is defined in a joint limit of large time $\tau$ and large system size $L$. In this work, we first take $\tau \rightarrow \infty$ and then take $L \rightarrow \infty$. The phase transition is first order, and the order parameter $K^{\infty}(s)=\lim _{L \rightarrow \infty}(1 / L)\langle K\rangle_{s}$ exhibits a discontinuous jump at $s=0$ [7]. However, the large- $L$ limit is not accessible numerically, and for finite $L$ the activity is a smooth function of $s$, whose representative behavior is shown in Fig. 1. The crossover sharpens as $L$ increases: to analyze the transition, one must consider the finite-size scaling of $\langle K\rangle_{s}$.

Cloning algorithm with feedback.-To perform this finite-size scaling, we require a numerical method that provides accurate results for a range of system sizes. To this end, we generalize a recently proposed adaptive method [30] to Markov jump processes. The method is based on a cloning algorithm $[19,33]$ that uses a population of $N_{c}$ clones (or copies) of the system. We fix a time interval $\Delta t$ and the dynamics of the model are propagated over intervals of length $\Delta t$, such that the total time is $\tau$. For each interval, one calculates a weighting factor for clone $a$ :

$$
\gamma_{a}=\exp \left\{-s\left[N_{a}^{K}(t+\Delta t)-N_{a}^{K}(t)\right]\right\},
$$

where $N_{a}^{K}(t)$ is the number of spin flips for clone $a$, evaluated over the whole time interval $[0, t]$; also one defines $K_{a}(t) \equiv N_{a}^{K}(t) / t$. After each time interval, clones are duplicated or removed, to enforce the conditioning on the activity. In this step, each clone $a$ generates a number of offspring proportional to its weight $\gamma_{a}$. The mean activity $\langle K\rangle_{s}$ can then be obtained as the average of $K_{a}(\tau)$ over the final population [30].

This algorithm provides accurate results when $N_{c}$ is sufficiently large [34], but in practice this may require a

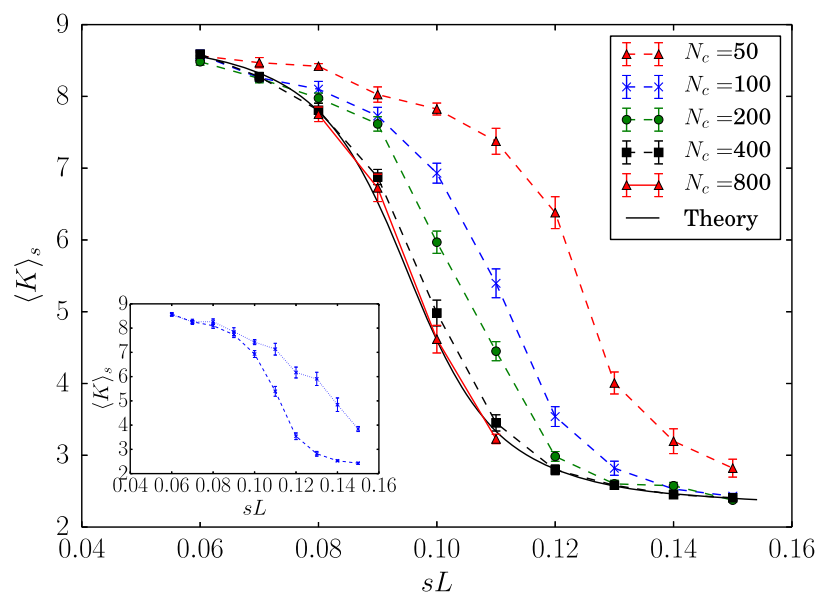

FIG. 1. The average activity $\langle K\rangle_{s}$ for $L=36$ as a function of $s L$, estimated using the feedback method described in the text. As the number of copies $N_{c}$ increases, the estimators of $\langle K\rangle_{s}$ converge to the correct result. The solid black line is the analytical form (11). The parameters $A, B, \kappa, s_{c}^{L}$ in Eq. (11) are determined by fitting the data outside of the coexistence region ( $s L=0.06-0.08,0.12-0.15$ ) for $N_{c}=400$, where the method converges rapidly. The inset shows a comparison between the results obtained from the feedback method (blue dashed line) and from the standard method (blue dotted line) for $N_{c}=100$.

very large number of clones, which is computationally expensive. To avoid this issue, we combine the existing cloning algorithm $[13,19-22,33,34]$ with a modification of the dynamics [35-38], following Ref. [30]. In order to aid sampling of trajectories with nontypical activity, we modify the transition rates of the model as

$w_{\text {mod }}\left(\mathcal{C} \rightarrow \mathcal{F}_{i}[\mathcal{C}]\right)=e^{-s} w\left(\mathcal{C} \rightarrow \mathcal{F}_{i}[\mathcal{C}]\right) e^{\frac{1}{2}\left[U(\mathcal{C})-U\left(\mathcal{F}_{i}[\mathcal{C}]\right)\right]}$,

where $U(\mathcal{C})$ is an effective potential or control potential [39]. The weight factors $\gamma_{a}$ are also modified, by replacing $-s N^{K}$ in Eq. (4) with

$$
K_{\text {mod }}=\int_{0}^{\tau} d t\left[k_{\text {mod }}\left(\mathcal{C}_{t}\right)-k\left(\mathcal{C}_{t}\right)\right],
$$

where $k(\mathcal{C})=\sum_{i} w\left(\mathcal{C} \rightarrow F_{i}[\mathcal{C}]\right)$ is the escape rate from configuration $\mathcal{C}$, and $k_{\text {mod }}$ is obtained in the same way but using the modified rates (5).

In the limit of large $N_{c}$, the results of the algorithm are independent of the choice of $U$. However, an appropriate choice can dramatically improve the accuracy of results obtained with finite populations. In particular, there exists an optimal control potential for which a population of $N_{c}=1$ is already sufficient for convergence. This optimal potential is given (up to an arbitrary constant) by $U^{*}(\mathcal{C})=2 \log \left[p_{\text {eq }}(\mathcal{C}) / p_{\text {end }}(\mathcal{C})\right]$, where $p_{\text {end }}(\mathcal{C})$ is the probability of observing configuration $\mathcal{C}$ within the steady state of the cloning algorithm [30]. Calculating this optimal control directly is not feasible in practice: instead we restrict to control potentials that involve interactions 
between each spin and its nearest neighbors at a distance $\leq d$, so that the change in the effective potential on flipping spin $i$ is

$$
U\left(\mathcal{F}_{i}[\mathcal{C}]\right)-U(\mathcal{C})=u_{d}\left(n_{i-d}, \ldots, n_{i}, \ldots, n_{i+d}\right)
$$

for some function $u_{d}$. To obtain the most suitable values for these potentials, we use a feedback scheme: we run the cloning algorithm, estimate the probabilities of particular local arrangements of the spins, and update the effective potential based on that choice. By repeating this procedure, one can optimize the choice of the control potential (see Refs. [30] and [40] for details).

Results.-Figure 1 shows the performance of the algorithm, close to the dynamical phase transition. We plot $\langle K\rangle_{s}$ as a function of $s L$, as obtained from the cloning algorithm, using the feedback method to determine suitable effective interactions. The interaction range is $d=4$, and we take $N_{c}$ between 50 and 800 . We set $\tau=15000$, which is sufficiently large to converge to the large- $\tau$ limit. As $N_{c}$ increases, the estimates of $\langle K\rangle_{s}$ converge to a smooth curve, indicating that these clone populations are large enough to achieve accurate results. By contrast, the inset to Fig. 1 shows that the original cloning method (with $U=0$ ) deviates significantly from the correct result, compared with the feedback method for the same number of copies. This tendency is observed throughout the whole range of $s$, irrespective of the presence of the dynamical coexistence [41].

The results of Fig. 1 show the expected crossover from high to low activity, consistent with the existence of a dynamical phase transition near $s=0$. To analyze the finite-size scaling of this transition, we define $\kappa$ as the maximal susceptibility

$$
\kappa \equiv \frac{1}{L^{2}} \max _{s}\left|\frac{\partial\langle K\rangle_{s}}{\partial s}\right| .
$$

Let the finite-size transition point $s_{c}^{L}$ be the value of $s$ at which this maximum occurs. For large systems, we expect $\kappa \rightarrow \infty$ and $s_{c}^{L}=\mathcal{O}\left(L^{-1}\right)$ [7,43]. Figure 2 shows the dependence of $\kappa$ on the system size $L$ : the results are consistent with an exponential divergence of $\kappa$ as $L \rightarrow \infty$, in contrast to traditional finite-size scaling at thermodynamic transitions, where $\kappa$ scales as a power of $L[24,31]$. To gain insight into these phase transitions and explain the numerical results in Figs. 1 and 2, we now present some theoretical arguments.

Analogy with a two-dimensional thermodynamic system on a cylinder.-These dynamical phase transitions in one dimension can be mapped to thermodynamic transitions in two dimensions (2D) [31,35]. Recalling that we have taken the limit $\tau \rightarrow \infty$ before taking $L \rightarrow \infty$, the relevant geometry for the $2 \mathrm{D}$ system is a long cylinder, with the system size $L$ in the dynamical system corresponding to the perimeter of the cylinder. For equilibrium systems in such

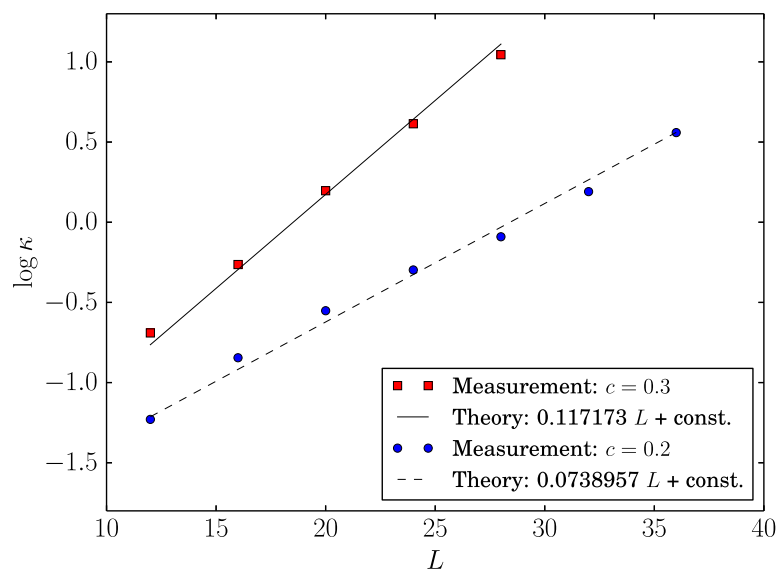

FIG. 2. Exponential divergence of the dynamical susceptibility $\kappa$, defined in Eq. (8). We plot $\log \kappa$ as a function of $L$, as obtained from the feedback method (points) together with the theoretical prediction (10) (straight lines).

geometries, the behavior near phase coexistence is sketched in Fig. $3[44,45]$ : the two phases form domains arranged along the cylinder. The typical domain length scales exponentially in $L$ : the reason is that these $2 \mathrm{D}$ domains are separated by domain walls of length $L$ that run around the cylinder, and the associated interfacial free-energy cost scales as $\alpha L$, so the density of domain walls is of order $\mathrm{e}^{-\alpha L}$. From Eq. (8), $\kappa$ is analogous to a susceptibility in the thermodynamic transition; it is also equal to a time integral of the autocorrelation function of $k\left(\mathcal{C}_{t}\right) / L$ [11]. Hence, $\kappa$ scales with the relaxation time of the system. Identifying this relaxation time with the domain size in Fig. 3, the susceptibility therefore diverges as $\kappa=O\left(e^{\alpha L}\right)$ for large $L$. The numerical data in Fig. 2 are consistent with such a divergence, indicating that the thermodynamic analogy can predict properties of the dynamical transition.

Effective interfacial model of the dynamical phase transition.-We now introduce a simplified effective model for the domains in Fig. 2. Following Sec. IV 1 of Ref. [46],

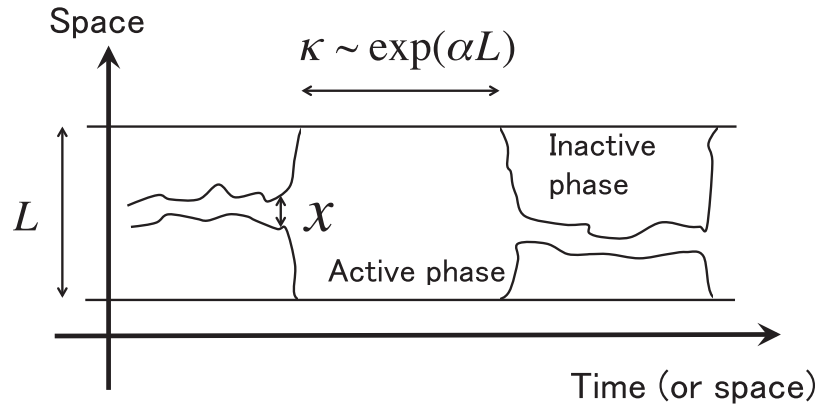

FIG. 3. Schematic picture of the domain wall dynamics at dynamical phase coexistence, which is analogous to equilibrium phase coexistence on the (2D) surface of a long cylinder $[44,45]$ (in this latter case the horizontal axis is a spatial coordinate). In the effective model, the fluctuating variable $x \in\{1,2, \ldots, L\}$ represents the width of the active phase. 
we assume that typical configurations in the model include a single active domain of size $x$ (Fig. 3). Within this domain, the system is close to its active (equilibrium) state; in the remainder of the system, the system is inactive and there are no up spins. The system contains at least one up spin so $1 \leq x \leq L$. We will show that this simplified model makes quantitatively accurate predictions for the dynamical phase transition. (For thermodynamic transitions, similar results may be available via spectral properties of the transfer matrix $[44,45]$ : our analysis here is different, and is based on the dynamical nature of the phase transition.)

The dynamical rules of the FA model mean that the value of $x$ increases with rate $2 c(1-c)$ and decreases with rate $2 c$ [46]. We take reflecting boundary conditions at $x=1$, $L$. [For systems that are predominately active, we interpret $(L-x)$ as the size of the largest inactive domain in the system, which has a typical value of order $1 / c$.] The activity in this effective model is obtained by assuming that the spins in the active domain flip with typical rate $\bar{k}=4 c^{2}(1-c)$, but there are no spin flips outside this region, due to the kinetic constraint. Hence, the analog of $K(\tau)$ is $(\bar{k} / \tau) \int_{0}^{\tau} x(t) d t$.

The result is an effective model where $x$ undergoes a random walk whose hop rates are biased to the right (positive $x$ ), but conditioned on a relatively small timeaveraged position. In the limit of large $L$, the model can be solved exactly, as shown in the Supplemental Material [47]. We summarize the main results: there is a dynamical phase transition at a field $s^{*}=\mu^{3} /(L \sqrt{\bar{k}})$, where $\mu$ solves

$$
\frac{1}{2} \mu^{3 / 2}+F(c)=\frac{4 c^{2}-\bar{k}+2 c \sqrt{\bar{k}} \Psi(c, \mu)}{4 c^{2}+\bar{k}-2 c \sqrt{\bar{k}} \Psi(c, \mu)} .
$$

Here, $F(c)=(1 / 2) \log [c /(1-c)]$ and $\Psi(c, \mu)=-\mu^{3}+$ $2[\cosh F(c)-1]$. Also, the susceptibility $\kappa$ diverges as

$$
\kappa \propto e^{\alpha L}, \quad \alpha=\frac{2}{3} \mu^{3 / 2} .
$$

Finally, the scaling form of the activity near the phase transition is

$$
\frac{\langle K\rangle_{s}}{L} \sim A-\frac{\kappa L\left(s-s_{c}^{L}\right)}{\sqrt{1+B \kappa^{2} L^{2}\left(s-s_{c}^{L}\right)^{2}}},
$$

where $A$ and $B$ are specified in Ref. [47]. This last result is similar to that obtained in a mean-field FA model [48] and that for $2 \mathrm{D}$ equilibrium phase coexistence of ferromagnets on a cylinder geometry [45]. These similarities indicate that the scaling function (11) might be a general property of firstorder phase transitions with exponentially diverging susceptibilities. We also remark that our effective model yields the interfacial free-energy cost $\alpha$ (9), (10), which is not available from the 2D equilibrium approach of Refs. [44,45].

The theoretical predictions (9)-(11) are shown in Figs. 1 and 2 , together with the numerical results. The agreement is excellent, despite the simplicity of the model. The conclusion is that the finite-size scaling of the phase transition is dominated by the dynamical properties of the interface between the active and inactive regions, and this interface is accurately described by the effective model. Moreover, in the analogy with the classical phase transition on a cylinder, we can interpret the parameter $\alpha$ in terms of an interfacial tension between the active and inactive domains shown in Fig. 2.

Discussion.-There are two key outcomes of this work. First, we have shown that the cloning-with-feedback algorithm used here allows accurate characterization of dynamical phase transitions for a range of system sizes, with much greater computational efficiency than the original cloning scheme. Second, we have shown how the finite-size scaling of first-order dynamical phase transitions can be understood qualitatively by mapping them to classical phase transitions in cylindrical geometries; it can also be analyzed quantitatively by mapping to the effective interfacial model.

We expect both the numerical and theoretical methods to apply generally for dynamical phase transitions of this type: for example, application to other kinetically constrained models [7,31] should be straightforward. We also anticipate application to atomistic systems that support similar phase transitions [13,16,20-22]. Moreover, the transitions considered here are directly related to quantum phase transitions in spin chains, for which results similar to Eq. (9) have been derived [49]. The effective interfacial model presented here provides a clear physical interpretation of such results, whose implications for quantum systems remain to be explored.

We also highlight several useful features of the numerical algorithm used here. The computational cost of the cloning algorithm scales linearly in the time $\tau$. This allows the large- $\tau$ limit to be converged numerically. Hence, the only parameter in the finite size scaling is $L$, which allows direct comparison with the theory presented here. This analysis is significantly simpler than finite-size scaling via path sampling, where both $\tau$ and $L$ must be varied together [13,21]. The cloning algorithm can also be applied in systems where detailed balance is broken, where path-sampling methods are not directly applicable. Cloning methods are also related to diffusion quantum Monte Carlo calculations [50]; it would be interesting to investigate how the cloning-with-feedback method might be applied in that context [51].

Another advantage of this method is that the control potential $U$ determined numerically provides physical insight into these dynamical phase transitions. In the active phase close to the transition, the control potential acts to suppress the number of up spins (reducing the activity), but one also finds an effective attraction between up spins [52]. This attraction is weak but decays slowly in space, which acts to stabilize the large spatial domains shown in Fig. 3 $[53,54]$. Based on the effective model, we expect that the optimal control potential $U^{*}$ should depend primarily on 
these domain sizes, so it naturally includes long-range interactions. It would be interesting to obtain a better understanding of optimal control potentials close to dynamical phase transitions, especially since incorporating such information into numerical methods now has the potential to significantly improve their performance. For example, one might consider transitions in other spin models [11] as well as exclusion processes [6,54,55]. In any case, we stress that while the ansatz (7) is much simpler than the optimal control, it still results in a significant improvement of the computational efficiency.

In conclusion, we have shown how a combination of numerical and theoretical methods provide a detailed insight into the dynamical phase transition in the FA model. The basic ideas of the method are quite general, such as the modification of the cloning algorithm with a feedback procedure to determine the optimal force, or the interfacial model as a coarse-grained description of systems near coexistence. For first-order dynamical transitions, we believe that effective interfacial models should apply rather generally. The numerical method has even broader potential application, although the choice of a suitable control potential will depend on the problem of interest-this remains to be explored.

T. N. gratefully acknowledges the support of Fondation Sciences Mathématiques de Paris_-EOTP NEMOT15RPO, PEPS LABS, and the LAABS Inphyniti Centre National de la Recherche Scientifique (CNRS) project. V. L. acknowledges support by ANR-15-CE40-0020-03 Grant LSD and by European Research Council (ERC) Starting Grant No. 680275 MALIG. T. N. and V.L. are grateful to G. Semerjian for discussions.

[1] J. Robert Dorfman, An Introduction to Chaos in Nonequilibrium Statistical Mechanics, Cambridge Lecture Notes in Physics Vol. 14 (Cambridge University Press, Cambridge, England, 1999).

[2] N. G. van Kampen, Stochastic Processes in Physics and Chemistry, 3rd ed. (Elsevier, Amsterdam, 2007).

[3] H. Hinrichsen, Non-equilibrium critical phenomena and phase transitions into absorbing states, Adv. Phys. 49, 815 (2000).

[4] M. R. Evans and T. Hanney, Nonequilibrium statistical mechanics of the zero-range process and related models, J. Phys. A 38, R195 (2005).

[5] L. Bertini, A. De Sole, D. Gabrielli, G. Jona-Lasinio, and C. Landim, Current Fluctuations in Stochastic Lattice Gases, Phys. Rev. Lett. 94, 030601 (2005).

[6] T. Bodineau and B. Derrida, Distribution of current in nonequilibrium diffusive systems and phase transitions, Phys. Rev. E 72, 066110 (2005).

[7] J. P. Garrahan, R. L. Jack, V. Lecomte, E. Pitard, K. van Duijvendijk, and F. van Wijland, Dynamical First-Order Phase Transition in Kinetically Constrained Models of Glasses, Phys. Rev. Lett. 98, 195702 (2007).
[8] H. Touchette, The large deviation approach to statistical mechanics, Phys. Rep. 478, 1 (2009).

[9] M. Merolle, J. P. Garrahan, and D. Chandler, Space-time thermodynamics of the glass transition, Proc. Natl. Acad. Sci. U.S.A. 102, 10837 (2005).

[10] R. L. Jack, J. P. Garrahan, and D. Chandler, Space-time thermodynamics and subsystem observables in a kinetically constrained model of glassy materials, J. Chem. Phys. 125, 184509 (2006).

[11] J. P. Garrahan, R. L. Jack, V. Lecomte, E. Pitard, K. van Duijvendijk, and F. van Wijland, First-order dynamical phase transition in models of glasses: An approach based on ensembles of histories, J. Phys. A 42, 075007 (2009).

[12] J. Kurchan, Six out of equilibrium lectures, arXiv:0901.1271.

[13] L. O. Hedges, R. L. Jack, J. P. Garrahan, and D. Chandler, Dynamic order-disorder in atomistic models of structural glass formers, Science 323, 1309 (2009).

[14] J. P. Garrahan, P. Sollich, and C. Toninelli, in Dynamical Heterogeneities in Glasses, Colloids, and Granular Media, edited by L. Berthier, G. Biroli, J.-P. Bouchaud, L. Cipelletti, and W. van Saarloos (Oxford University Press, New York, 2011).

[15] D. Chandler and J. P. Garrahan, Dynamics on the way to forming glass: Bubbles in space-time, Annu. Rev. Phys. Chem. 61, 191 (2010).

[16] D. T. Limmer and D. Chandler, Theory of amorphous ices, Proc. Natl. Acad. Sci. U.S.A. 111, 9413 (2014).

[17] T. Bodineau and B. Derrida, Cumulants and large deviations of the current through non-equilibrium steady states, C.R. Phys. 8, 540 (2007).

[18] L. Bertini, A. De Sole, D. Gabrielli, G. Jona-Lasinio, and C. Landim, Non equilibrium current fluctuations in stochastic lattice gases, J. Stat. Phys. 123, 237 (2006).

[19] C. Giardinà, J. Kurchan, and L. Peliti, Direct Evaluation of Large-Deviation Functions, Phys. Rev. Lett. 96, 120603 (2006).

[20] E. Pitard, V. Lecomte, and F. Van Wijland, Dynamic transition in an atomic glass former: A molecular dynamics evidence, Europhys. Lett. 96, 56002 (2011).

[21] T. Speck and D. Chandler, Constrained dynamics of localized excitations causes a non-equilibrium phase transition in an atomistic model of glass formers, J. Chem. Phys. 136, 184509 (2012)

[22] T. Speck, A. Malins, and C. Patrick Royall, First-Order Phase Transition in a Model Glass Former: Coupling of Local Structure and Dynamics, Phys. Rev. Lett. 109, 195703 (2012).

[23] V. Privman and M. E. Fisher, Finite-size effects at first-order transitions, J. Stat. Phys. 33, 385 (1983).

[24] C. Borgs and R. Kotecký, A rigorous theory of finite-size scaling at first-order phase transitions, J. Stat. Phys. 61, 79 (1990).

[25] B. A. Berg and T. Neuhaus, Multicanonical Ensemble: A New Approach to Simulate First-Order Phase Transitions, Phys. Rev. Lett. 68, 9 (1992).

[26] F. Wang and D. P. Landau, Efficient, Multiple-Range Random Walk Algorithm to Calculate the Density of States, Phys. Rev. Lett. 86, 2050 (2001).

[27] A. D. Bruce and N. B. Wilding, Computational strategies for mapping equilibrium phase diagrams, Adv. Chem. Phys., 127, 1 (2003). 
[28] T. R. Gingrich and P. L. Geissler, Preserving correlations between trajectories for efficient path sampling, J. Chem. Phys. 142, 234104 (2015).

[29] G. H. Fredrickson and H. C. Andersen, Kinetic Ising Model of the Glass Transition, Phys. Rev. Lett. 53, 1244 (1984).

[30] T. Nemoto, F. Bouchet, R. L. Jack, and Vivien Lecomte, Population-dynamics method with a multicanonical feedback control, Phys. Rev. E 93, 062123 (2016).

[31] Y. S. Elmatad, R. L. Jack, D. Chandler, and J. P. Garrahan, Finite-temperature critical point of a glass transition, Proc. Natl. Acad. Sci. U.S.A. 107, 12793 (2010).

[32] F. Ritort and P. Sollich, Glassy dynamics of kinetically constrained models, Adv. Phys. 52, 219 (2003).

[33] C. Giardinà, J. Kurchan, V. Lecomte, and J. Tailleur, Simulating rare events in dynamical processes, J. Stat. Phys. 145, 787 (2011).

[34] T. Nemoto, E. G. Hidalgo, and V. Lecomte, Finite-time and finite-size scalings in the evaluation of large-deviation functions: Analytical study using a birth-death process, Phys. Rev. E 95, 012102 (2017).

[35] R. L. Jack and P. Sollich, Large deviations and ensembles of trajectories in stochastic models, Prog. Theor. Phys. Suppl. 184, 304 (2010).

[36] V. Popkov, G. M. Schütz, and D. Simon, ASEP on a ring conditioned on enhanced flux, J. Stat. Mech. (2010) P10007.

[37] R Chetrite and H. Touchette, Nonequilibrium Microcanonical and Canonical Ensembles and Their Equivalence, Phys. Rev. Lett. 111, 120601 (2013).

[38] T. Nemoto and S.-ichi Sasa, Computation of Large Deviation Statistics via Iterative Measurement-and-Feedback Procedure, Phys. Rev. Lett. 112, 090602 (2014).

[39] R. L. Jack and P. Sollich, Effective interactions and large deviations in stochastic processes, Eur. Phys. J. Spec. Top.224 2351 (2015).

[40] See Supplemental Material at http://link.aps.org/ supplemental/10.1103/PhysRevLett.118.115702 for the details of the feedback algorithm, first section.

[41] See Supplemental Material at http://link.aps.org/ supplemental/10.1103/PhysRevLett.118.115702 for numerical evidence supporting the feedback algorithm compared to the standard one, second section, which includes Ref. [42].
[42] E. G. Hidalgo, T. Nemoto, and V. Lecomte, Finite-time and -size scalings in the evaluation of large deviation functions_-part II: Numerical approach in continuous time, arXiv:1607.08804.

[43] T. Bodineau and C. Toninelli, Activity phase transition for constrained dynamics, Commun. Math. Phys. 311, 357 (2012).

[44] V. Privman and M. E. Fisher, Finite-size effects at first-order transitions, J. Stat. Phys. 33, 385 (1983).

[45] C. Borgs and J. Z. Imbrie, Crossover finite-size scaling at first-order transitions, J. Stat. Phys. 69, 487 (1992).

[46] T. Bodineau, V. Lecomte, and C. Toninelli, Finite size scaling of the dynamical free-energy in a kinetically constrained model, J. Stat. Phys. 147, 1 (2012).

[47] See Supplemental Material at http://link.aps.org/ supplemental/10.1103/PhysRevLett.118.115702 for the derivation of Eqs. (9), (10) and (11), third section.

[48] T. Nemoto, V. Lecomte, S. Sasa, and F. van Wijland, Finitesize effects in a mean-field kinetically constrained model: Dynamical glassiness and quantum criticality, J. Stat. Mech. (2014) P10001.

[49] M. Heyl, A. Polkovnikov, and S. Kehrein, Dynamical Quantum Phase Transitions in the Transverse-Field Ising Model, Phys. Rev. Lett. 110, 135704 (2013).

[50] J. B. Anderson, A randomwalk simulation of the Schrödinger equation: $\mathrm{H}_{3}^{+}$, J. Chem. Phys. 63, 1499 (1975).

[51] M. Casula, Beyond the locality approximation in the standard diffusion Monte Carlo method, Phys. Rev. B 74, 161102 (2006).

[52] See Supplemental Material at http://link.aps.org/ supplemental/10.1103/PhysRevLett.118.115702 for a study of the effective interactions around the dynamical phase transition, fourth section.

[53] R. L. Jack and P. Sollich, Large deviations of the dynamical activity in the East model: Analysing structure in biased trajectories, J. Phys. A 47, 015003 (2014).

[54] R. L. Jack, I. R. Thompson, and P. Sollich, Hyperuniformity and Phase Separation in Biased Ensembles of Trajectories for Diffusive Systems, Phys. Rev. Lett. 114, 060601 (2015).

[55] V. Lecomte, J. P. Garrahan, and F. van Wijland, Inactive dynamical phase of a symmetric exclusion process on a ring, J. Phys. A 45, 175001 (2012). 